\title{
Surgical Excision of a Rare Case of Mammary Analogue Secretory Carcinoma: A Case Review
}

\author{
Elias Fakhoury ${ }^{\mathrm{a}}$, Sundus Abbasi ${ }^{\mathrm{a}}$, Shauna Trinh" ${ }^{\mathrm{a}, \mathrm{b}, \mathrm{c}}$, Mark Connolly, \\ Ronald Jason Pope ${ }^{\mathrm{a}}$
}

\begin{abstract}
Mammary analogue secretory carcinoma (MASC) is a newly recognized salivary gland neoplasm that shares characteristics with secretory carcinoma of the breast. Since its first description by Skalova et al in 2010, many reviews have been published. While current research has described specific immunohistochemistry and genetic translocations of MASC, there is much in terms of management that remains unknown. We present a unique case of a 20 -year-old male with a parotid tumor that was reclassified as MASC based on its histologic and immunohistochemical findings, and we describe our management.
\end{abstract}

Keywords: Mammary analogue secretory carcinoma; ETV6-NTRK3 fusion gene; Salivary gland tumor

\section{Introduction}

With the recent recognition of mammary analogue secretory carcinoma (MASC), many previously diagnosed acinic cell carcinomas and adenocarcinomas not otherwise specified (NOS) have been reclassified to MASC based on specific histologic, immunohistochemical, and genetic findings. MASC is a newly described salivary gland neoplasm characterized by an ETV6 translocation and shares histologic and immunohistochemical findings similar to secretory carcinoma of the breast [1]. In this case report, we discuss the rare case of MASC in a 20-year-old male with a parotid tumor.

\section{Case Report}

A 20-year-old male with no significant past medical history

\section{Manuscript accepted for publication December 28, 2015}

aDepartment of Surgery, Saint Joseph's Regional Medical Center, 703 Main Street, Paterson, NJ 07503, USA

bNew York Medical College, 40 Sunshine Cottage Rd, Valhalla, NY 10595, USA

'Corresponding Author: Shauna Trinh, Department of Surgery, Saint Joseph's Regional Medical Center, 703 Main Street, Paterson, NJ 07503, USA.

Email: shauna.trinh@gmail.com

doi: http://dx.doi.org/10.14740/jcs291w presented with a 3-year history of a left-sided face mass along the angle of the jaw, which he stated had progressively increased in size. The patient denied tenderness, fever, chills, or any recent weight loss. The mass was solid, mobile, and measured approximately $2 \times 2 \mathrm{~cm}$ in size. A volumetric computed tomography $(\mathrm{CT})$ scan of the neck demonstrated a centrally fluid-attenuated cystic structure measuring $2.2 \times 2.2 \times 2.1 \mathrm{~cm}$ in the tail of the parotid gland (Fig. 1). A formal excision of the cystic structure was performed, and no pathological nodes were detected in the neck. The tumor was ultimately classified as an MASC of the salivary gland. Postoperative radiotherapy was not recommended, and at an in-office follow-up, the patient remained without evidence of disease.

\section{Discussion}

MASC is a newly recognized salivary gland neoplasm first characterized in 2010 that shares similar histopathology and genetics with secretory carcinoma of the breast. MASC expresses the $\mathrm{t}(12 ; 15)(\mathrm{p} 13 ; \mathrm{q} 25)$ translocation resulting in the

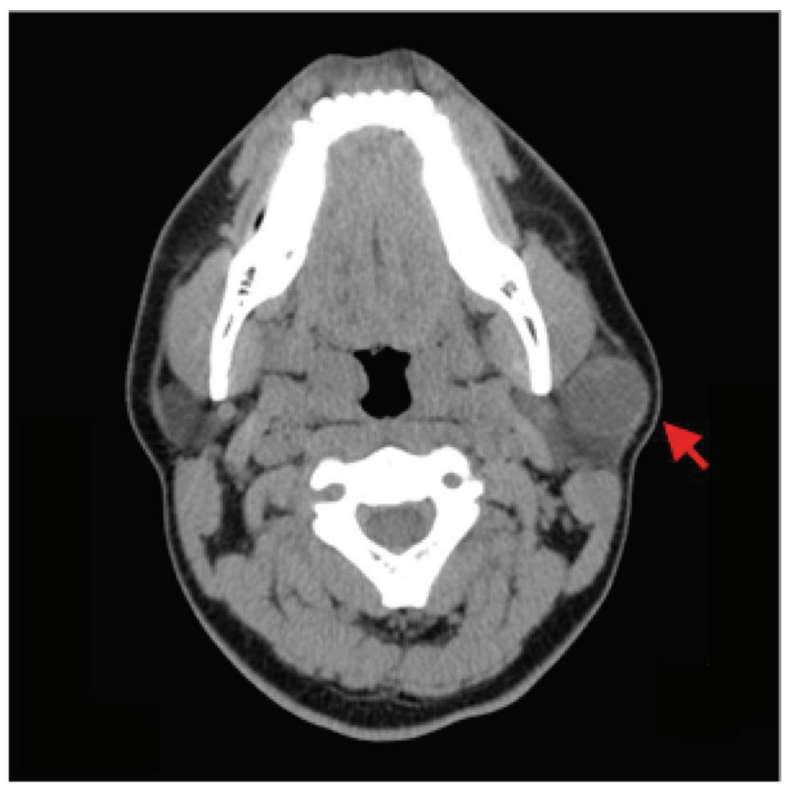

Figure 1. Computed tomography (CT) of the neck. Volumetric noncontrast CT of the soft tissues of the neck demonstrates a well-circumscribed $2.2 \times 2.2 \times 2.1 \mathrm{~cm}$ cystic tumor in the tail of the left parotid gland (red arrow). 


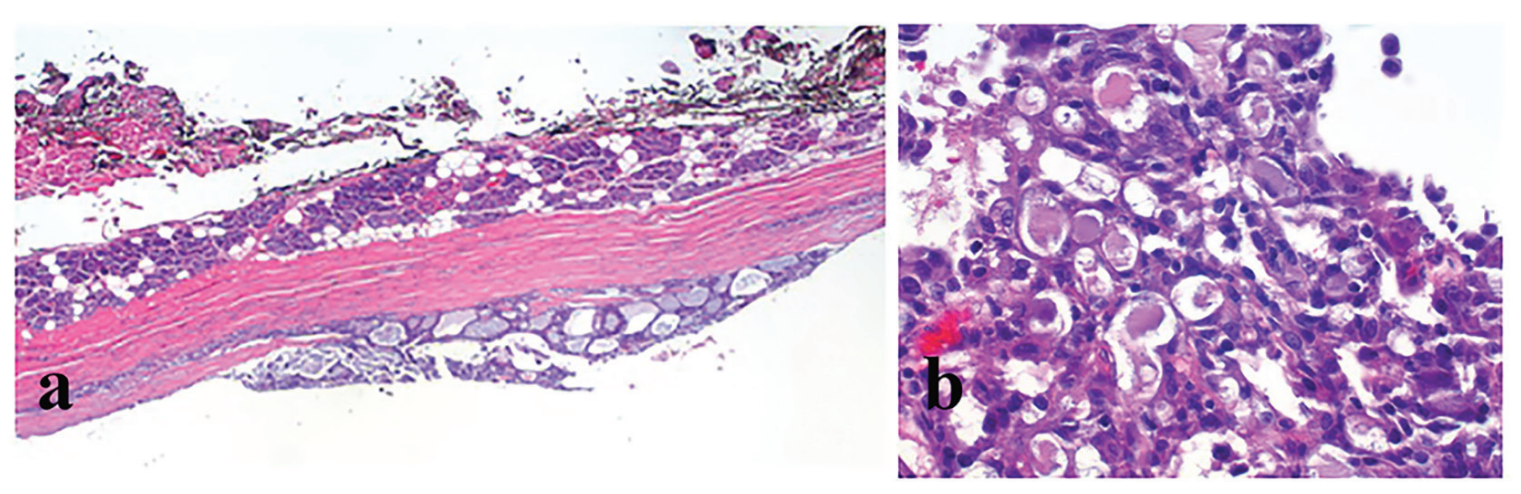

Figure 2. H\&E stain. (a) Low-powered view demonstrates a well-circumscribed, cystic epithelial neoplasm (bottom) that is set apart from normal serous glandular elements of the adjacent parotid (top) by a pauci-cellular fibrous capsule. (b) High-powered view demonstrates an epithelial neoplasm with a microcystic pattern of growth. There is presence of extracellular eosinophilic mucin secretions.

ETV6-NTRK3 fusion gene, which potentiates tyrosine kinase activity and promotes oncogenesis [2-4]. MASC is commonly a solitary, well-circumscribed, encapsulated mass located in the parotid gland, but it may also develop from the oral cavity, submandibular gland, or the accessory parotid glands [3, 5]. It is commonly detected incidentally on physical examination and presents as a slowly enlarging and painless nodule [3]. One patient was found to have facial paralysis with bulky growth of a parotid gland tumor [3]. Previously, MASC was classified with other low-grade salivary carcinomas, most commonly mislabeled as acinic cell carcinoma (ACC) or ADC-NOS $[1,3]$. Unlike ACC, MASC has no gender preference, a mean incidence age of 40, and the potential for lymph node metastasis [3]. Additionally, MASC is reactive to S100 protein and mammaglobin and unreactive to DOG1 $[1,6]$. Other histological features of MASC include cystic, tubular, and/or papillary architecture, eosinophilic vacuolated cytoplasm, intraluminal or intracellular colloid-like secretions, and pale nuclei [3].

Histopathology of our patient's parotid mass revealed a cystic lesion lined with cuboidal to columnar epithelium (Fig. $2 \mathrm{a}, \mathrm{b})$. There was a focus of cystic proliferation surrounded by a fibrous wall. The lumen and the wall of the cyst focally contained hemorrhagic material and foam cells. From the histopathological findings, the differential diagnoses included cystadenoma of the parotid gland, salivary duct cyst with hyperplasia, and duct ectasia with focal epithelial proliferation. Immunohistochemistry was subsequently performed, and the tumor was found to be regionally positive for S100 protein and mammaglobin and negative for DOG1. The parotid tumor was diagnosed as MASC, further emphasizing the role that histology and immunohistochemistry play in the recognition of MASC. Fluorescence in situ hybridization (FISH) for ETV6 gene rearrangement, characteristic of MASC, can be used as a supplement but it is not necessary nor definitive for diagnosis $[3,7]$.

Standard of care for low-grade malignant salivary gland cancers is surgical resection. Due to limited information regarding the prognostic behavior of MASC, the benefits of neck dissection and postoperative radiotherapy are still under con- sideration [3].

\section{Conclusion}

Since the first description of MASC in 2010, only 92 cases have been reported, highlighting this newly classified carcinoma $[3,5]$. In addition, $19 \%$ of parotid gland tumors and $79 \%$ of extra-parotid gland tumors originally classified as ACC were reclassified as MASC by Bishop [5]. While there are specific immunohistochemical and genetic characteristics specific to MASC, the clinical behavior, prognosis, and treatment of this analogue are limited.

\section{References}

1. Skalova A, Vanecek T, Sima R, Laco J, Weinreb I, PerezOrdonez B, Starek I, et al. Mammary analogue secretory carcinoma of salivary glands, containing the ETV6NTRK3 fusion gene: a hitherto undescribed salivary gland tumor entity. Am J Surg Pathol. 2010;34(5):599-608.

2. Chi HT, Ly BT, Kano Y, Tojo A, Watanabe T, Sato Y. ETV6-NTRK3 as a therapeutic target of small molecule inhibitor PKC412. Biochem Biophys Res Commun. 2012;429(1-2):87-92.

3. Sethi R, Kozin E, Remenschneider A, Meier J, VanderLaan P, Faquin W, Deschler D, et al. Mammary analogue secretory carcinoma: update on a new diagnosis of salivary gland malignancy. Laryngoscope. 2014;124(1):188-195.

4. Stenman G. Fusion oncogenes in salivary gland tumors: molecular and clinical consequences. Head Neck Pathol. 2013;7(Suppl 1):S12-19.

5. Bishop JA. Unmasking MASC: bringing to light the unique morphologic, immunohistochemical and genetic features of the newly recognized mammary analogue secretory carcinoma of salivary glands. Head Neck Pathol. 2013;7(1):35-39.

6. Takeda M, Kasai T, Morita K, Takeuchi M, Nishikawa T, Yamashita A, Mikami S, et al. Cytopathological features 
of mammary analogue secretory carcinoma - review of literature. Diagn Cytopathol. 2015;43(2):131-137.

7. Shah AA, Wenig BM, LeGallo RD, Mills SE, Stelow EB.
Morphology in conjunction with immunohistochemistry is sufficient for the diagnosis of mammary analogue secretory carcinoma. Head Neck Pathol. 2015;9(1):85-95. 